\title{
Improvements in short-term forecasting of geomagnetic activity
}

\author{
Ramkumar Bala ${ }^{1}$ and Patricia Reiff ${ }^{1}$ \\ Received 15 February 2012; revised 5 April 2012; accepted 16 April 2012; published 6 June 2012.
}

[1] We have improved our space weather forecasting algorithms to now predict Dst and $A E$ in addition to $K p$ for up to $6 \mathrm{~h}$ of forecast times. These predictions can be accessed in real time at http://mms.rice. edu/realtime/forecast.html. In addition, in the event of an ongoing or imminent activity, e-mail "alerts" based on key discriminator levels have been going out to our subscribers since October 2003. The neural network-based algorithms utilize ACE data to generate full 1, 3, and $6 \mathrm{~h}$ ahead predictions of these indices from the Boyle index, an empirical approximation that estimates the Earth's polar cap potential using solar wind parameters. Our models yield correlation coefficients of over $0.88,0.86$, and 0.83 for $1 \mathrm{~h}$ predictions of $K p$, Dst, and $A E$, respectively, and $0.86,0.84$, and 0.80 when predicting the same but $3 \mathrm{~h}$ ahead. Our $6 \mathrm{~h}$ ahead predictions, however, have slightly higher uncertainties. Furthermore, the paper also tests other solar wind functions-the Newell driver, the Borovsky control function, and adding solar wind pressure term to the Boyle index-for their ability to predict geomagnetic activity.

Citation: Bala, R., and P. Reiff (2012), Improvements in short-term forecasting of geomagnetic activity, Space Weather, 10, S06001, doi:10.1029/2012SW000779.

\section{Introduction}

[2] This paper summarizes our efforts at the Rice Space Institute to provide short- and long-term forecasts of geomagnetic activity, namely $K p$, Dst, and $A E$, to provide a faster alert system than the official nowcasts from the Space Weather Prediction Center at NOAA. This is an extension in forecast time and in predicted parameters from our previous paper [Bala et al., 2009, hereinafter paper 1]. We use solar wind-magnetosphere coupling functions, which represent the dayside merging rates in terms of solar wind parameters, as basis functions to train an artificial neural network (ANN). Several studies in the past [e.g., Kamide et al., 1998; Papitashvili et al., 2000; Newell et al., 2007] have underscored the benefits of applying this concept of coupling functions to space weather, making them good candidates to describe the state of the magnetosphere system over a wide variety of magnetospheric activity (e.g., Dst, $A E, K p$, Auroral Power, Polar cap size, b2i, AL, etc.).

[3] The Boyle index (BI), $\Phi(k V)=10^{-4}\left(\frac{v}{k m / s e c}\right)^{2}+$ $11.7\left(\frac{B}{n T}\right) \sin ^{3}(\theta / 2)$, is an empirically derived scalar function that approximates the steady state polar cap potential

\footnotetext{
${ }^{1}$ Department of Physics and Astronomy, William Marsh Rice University, Houston, Texas, USA.

Corresponding author: R. Bala, Department of Physics and Astronomy, William Marsh Rice University, 6100 Main St., Houston, TX 77005, USA. (ramkumar@rice.edu)
}

(PCP), where $v$ is the solar wind velocity in $\mathrm{km} / \mathrm{sec}$, $\mathrm{B}$ is the magnitude of the IMF in nanoteslas, and $\theta$ is the clock angle [Boyle et al., 1997]. PCP is one of the fundamental measures of the coupling rate between the solar wind and the Earth's magnetosphere. Thus, although the BI was derived only to predict the PCP, it is reasonable to use it as a possible coupling function to "forecast" other measures of geomagnetic activity (e.g., $K p$, the ring current Dst index, and the auroral electrojet index $A E$ ) because geomagnetic indices can be modeled using solar wind derivatives.

[4] Our models to predict the $K p$ index from the BI using ANNs have been discussed previously in paper 1 . In this work, besides providing an updated version of our $\mathrm{Kp}$ models, we are also introducing new models that can predict Dst and $A E$. Part of the job of a space weather forecaster is to aid the process of decision making during critical times and to make the available space weather information more useful. In order to achieve this goal, one would want to forecast for different lead times. Here, we choose to forecast with 1,3 , and $6 \mathrm{~h}$ lead times. The purpose of a $1 \mathrm{~h}$ ahead prediction is to provide a short-term warning to certain end users for whom such a warning can be of benefit; for example, satellite operators, electrical transmission line companies. In addition, the effectiveness of the BI in raising the baseline further in short-term geomagnetic activity index forecasting is investigated and whether legitimate forecasting is plausible beyond lead times of over $3 \mathrm{~h}$, perhaps up to $6 \mathrm{~h}$. A $6 \mathrm{~h}$ predicted $K p$, $D s t$, and $A E$ will be regarded as a new baseline for further 
studies since most of the best estimates available in the literature are delivered for the next 2 to $4 \mathrm{~h}$ from the predicted time.

[5] For this purpose, we have developed fully automated time predictive algorithms using ANNs with prediction capabilities for moderate (e.g., $-40 \mathrm{nT}<D s t<-80 \mathrm{nT}$ ) to severe storms (e.g., Dst $<-120 \mathrm{nT}, K p>6$ ) in near real time. We have trained ANNs that take only solar wind and magnetospheric data from ACE to forecast in 3 different modes: (1) $1 \mathrm{~h}$ ahead, (2) $3 \mathrm{~h}$ ahead, and (3) $6 \mathrm{~h}$ ahead. For example, at time $\sim 0307 \mathrm{UT}$, the solar wind data up to 0300 UT become available and, mode 1 predicts the expected hourly values of $K p$, Dst, and $A E$ for 0300-0359 UT; mode 2 for 0500-0559 UT for Dst and AE and for 0300$0559 \mathrm{UT}$ for $\mathrm{Kp}$; and mode 3 for $0600-0859 \mathrm{UT}$ as $3 \mathrm{~h}$ averages of $K p$, Dst, and $A E$.

\section{Data and Training}

[6] The BIs and the geomagnetic indices used to train the ANNs comes from the archived data from the OMNIWeb (http://omniweb.gsfc.nasa.gov/ow.html), of the SPDF at Goddard Space Flight Center. The data range used in this study covers 1998-2009 for a complete solar cycle and is low resolution that has been time shifted to provide "at Earth" measurements of $1 \mathrm{~h}$ averages. With copious data available for this study ( $>100,000$ points), a good approach to building a steady neural network is to judiciously stratify the data into three smaller but distinct samples, one for "training", one for "testing" the error and the other for "validating" the model. Testing set is used exclusively during the network learning procedure to prevent the network from over or under fitting the data. Validation data are used to evaluate models and are not part of either the training or the testing set. In this study, data covering January 1998 to March 2001, May 2001 to December 2005, and January 2008 to December2009 were chosen for training ( $49 \%$ as $1 \mathrm{~h}$ and $59 \%$ as $3 \mathrm{~h}$ averages) and testing ( $31 \%$ as $1 \mathrm{~h}$ and $22 \%$ as $3 \mathrm{~h}$ averages), while the data from April 2001 and from January 2006 to December 2007 were used only for validating the models.

[7] The architecture and training techniques follows paper 1. In this work, however, inputs to the network follows a simple rule that the most recent value of the solar wind input receives the maximum weight $(w)$ of unity, the second most recent value receiving $0.9(0<w<1)$ of the previous, and so on $\left(w^{n}, n=0,1,2, \ldots\right)$. This procedure is an arbitrary choice, and the rationale behind this scheme of weighting the inputs is based on the assumption that the most recent solar wind values contribute the most to the predicted results. The network training algorithm, however, will internally adjust the final weighting of the terms.

\section{The BI and Space Weather}

[8] The BI was launched into real-time mode in October 2003, purely for a scientific and educational motive. It is available from http://mms.rice.edu/realtime/forecast.html as a courtesy of the Rice Space Institute and the NASA MMS mission. Since its inception, the subscribers to the "spacalrt" mailing list receive e-mail notices whenever the 10 min BI average exceeds $200 \mathrm{kV}$, called "red alerts." In over 8 years of real-time operations, it has enjoyed a lot of success and no major storm $(K p>6)$ has been missed, which demonstrates its value as a forecasting tool. In conjunction with the BI (asymptotic polar cap potential) real-time plot, the two $K p$ prediction models discussed in paper 1 ( 1 and $3 \mathrm{~h} \mathrm{BI}$ only) have culminated into real-time space weather forecast systems already; we have been running these two purely solar wind-driven models since October 2007 with good success.

[9] The BI is an actual predictor of the polar cap potential only if the steady state conditions prevail in the IMF for at least $4 \mathrm{~h}$, whereas this whole study is based on 1 and $3 \mathrm{~h}$ averages of the BI. However, by setting up a nonlinear neuron model through ANNs, we can capture the effects of non-steady states in the solar wind by weighting timeintegrated BI values over time, i.e., looking back several hours into the past. This is particularly useful to study the effects of short-term versus extended drivers, which tend to be nonlinear. To be a more accurate predictor of the polar cap potential during strong events, one should add a saturation term to the BI, since the observed potential typically saturates during times of strong solar wind activity [Hairston et al., 2003; Siscoe et al., 2002]. However, our findings indicate that the full $\mathrm{BI}$ (without a saturation term) is an excellent predictor of $K p, D s t$, and $A E$ in both moderate and severe conditions; therefore, although the polar cap potential saturates, the magnetospheric response does not. This could be vital for an operational setup, setting limits and thresholds for alerts, etc. This study and paper 1 are unique in that they are the first ANN prediction models to use only a single input (a coupling function) or, at the most, two inputs of the solar wind functions. These claims and the details of our ANN predictions will be discussed later in the paper.

[10] Our efforts earlier at the Rice Space Institute is outlined in Figure 1. It illustrates actual "forecast" results published in near real time using the previous version of the models. As the new solar cycle 24, which started in December 2008, started to ramp up, it threw a surprise on 22 July 2009 when the $1 \mathrm{~h}$ BI (Figure 1, first panel) almost reached $200 \mathrm{kV}$ for 2 full hours and the corresponding $3 \mathrm{~h}$ BI exceeded $160 \mathrm{kV}$. The success of our $1 \mathrm{~h}$ model predictions is shown in Figure 1 (fourth panel) as "white" histograms against "black" histograms, which represents the measured $1 \mathrm{~h} \mathrm{Kp}$ oversampled over the same interval. The overall statistics of the $1 \mathrm{~h}$ model for the time interval shown in Figure 1 are $r=0.859$, RMS error $=0.81$. Correspondingly, the activity was also predicted well by our $3 \mathrm{~h}$ BI model (depicted in the second and third panels). While the second panel compares the model 2 predictions against the NOAA/SWPC nowcast $K p(r=0.836$ and RMS error $=0.768$ overall), the third panel compares the official Kps against our model predictions $(r=0.842$ and RMS 

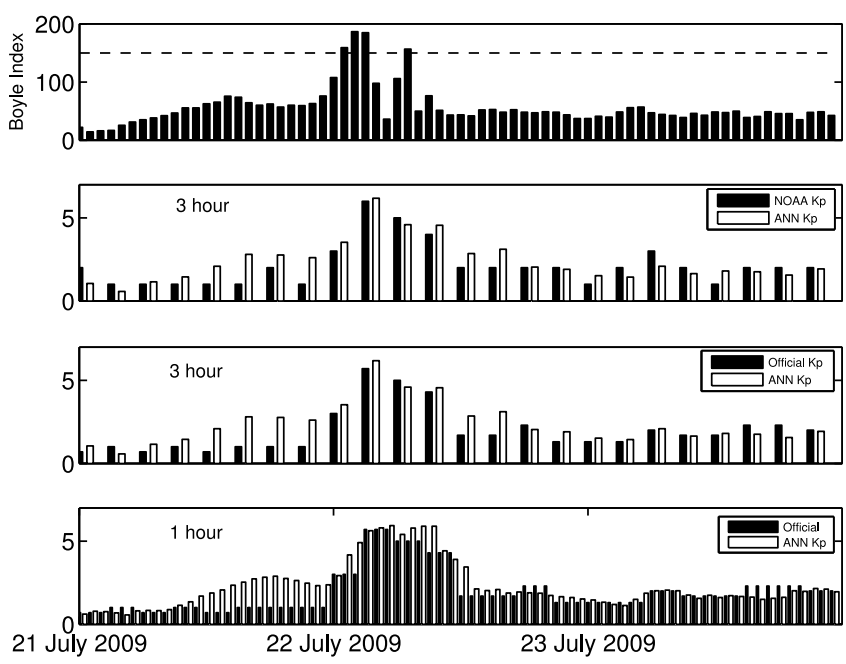

Figure 1. Results of our BI-only model $K p$ predictions from an activity (22 July 2009).

error $=0.843$ overall). The most critical aspect of any prediction algorithm lies in its ability to predict the timing of the onset. In this case the onset of the activity, demonstrated by $\Delta K p=+3$, and the resulting high $K p s(>4)$, were both well predicted by the network approximately $3 \mathrm{~h}$ ahead.

[11] The NOAA/SWPC derives $3 \mathrm{~h}$ planetary $K p$ index in near real time (http://www.swpc.noaa.gov/rt_plots/ $\left.\mathrm{kp} \_3 d . h t m l\right)$ using data from ground-based magnetometers, 5 sites in the USA and an observatory each located in UK and France. They report their final real time and best estimates of $K p$ at the end of the prescribed $3 \mathrm{~h}$ interval $(0000-0300,0300-0600, \ldots \ldots)$, thus providing a significant improvement for space weather forecasting over the monthly official releases. However, the limitation is that these are low-resolution $K p$, approximated to the nearest integer values $(0,1, \ldots, 9)$.

\section{Related Work}

[12] Several algorithms have been developed to nowcast $K p$ [e.g., Takahashi et al., 2001] and forecast $K p, D s t$, and $A E$ [e.g., Costello, 1997; Takalo and Timonen, 1997; Gleisner and Lundstedt, 1997; Boberg et al., 2000; Wing et al., 2005]. The ANN-based Wing et al. [2005] APL models have been deployed by the NOAA to take ACE data and generate $K p$ every 15 min with an early warning of up to $4 \mathrm{~h}$ in advance. Lundstedt and Wintoft [1994] and $W u$ and Lundstedt [1997] have performed early work to train ANNs to come up with predictions of the Dst index. Lindsay et al. [1999] were able to model Dst using solar wind monitors aboard different spacecraft inside $1 \mathrm{AU}$. Furthermore, Boaghe et al. [2001] came up with a Dst forecast model based on a nonlinear technique (NARMAX). Temerin and $L i[2002,2006]$ provided a new model for the Dst based on the solar wind parameters through rigorous empirical studies. Algorithms to predict $A E$ through ANNs include Gleisner and Lundstedt [2001], Gavrishchaka and Ganguli [2001], and, more recently, Pallocchia et al. [2008]. A computationally optimized nonlinear dynamical model of the magnetosphere-ionosphere system called the WINDMI [Horton et al., 2005] has been used to evaluate geomagnetic storms that have also focused on the prediction of the westward auroral electrojets (AL) and Dst indices.

\section{Prediction Algorithms}

[13] The $K p, D s t$, and $A E$ indices respond on varying time scales for a given set of solar wind parameters. The BI can be used to characterize the magnetospheric activity over these three distinct ground-based geomagnetic indices [e.g., Bala et al., 2009; Balasubramanian, 2010]. In order to obtain the best predictions, we sought out to find the optimum "look back" time for the best predictive power; i.e., how much of the solar wind history is needed in order to obtain the best prediction. The illustration shown in Figure 2 is a test of ANN "sensitivity" as a function of time or the number of hours of inputs. It shows that the correlation with the $A E$ index does not improve with additional hours of "look back" time, thus it is more a "directly driven" activity index. In contrast, however, the Kp and Dst predictions improve significantly with the addition of 6 or more hours of previous time history of solar wind input.

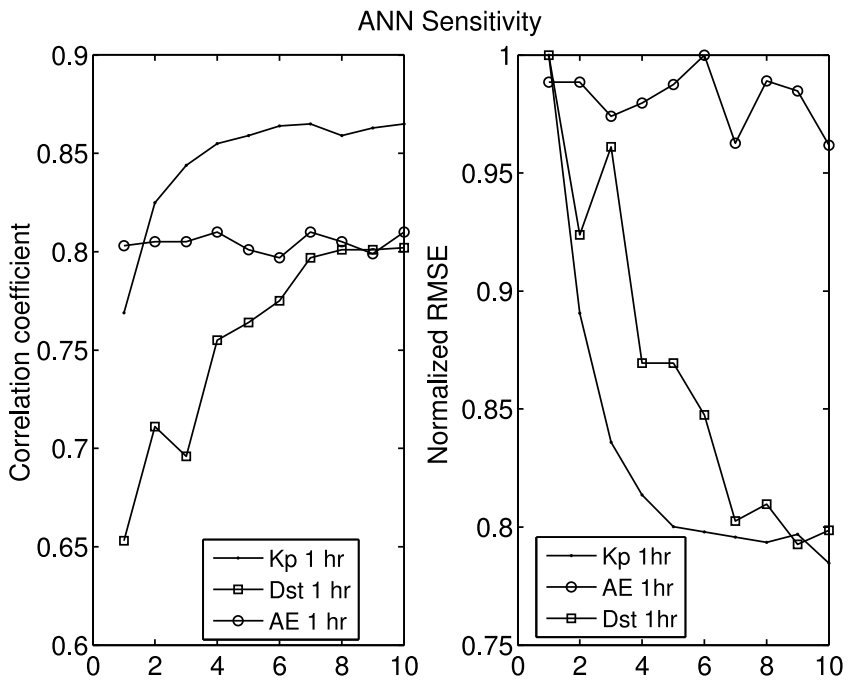

Figure 2. ANN sensitivity test, the correlation coefficient as a function of number of hours of past observations included in the training. For the $K p$ and Dst, the number of inputs with extended time history enhances the network performance before saturating around six or seven inputs. However, for the $A E$, the function asymptotes almost immediately; i.e., it is best predicted by just a few previous hours of IMF data. 

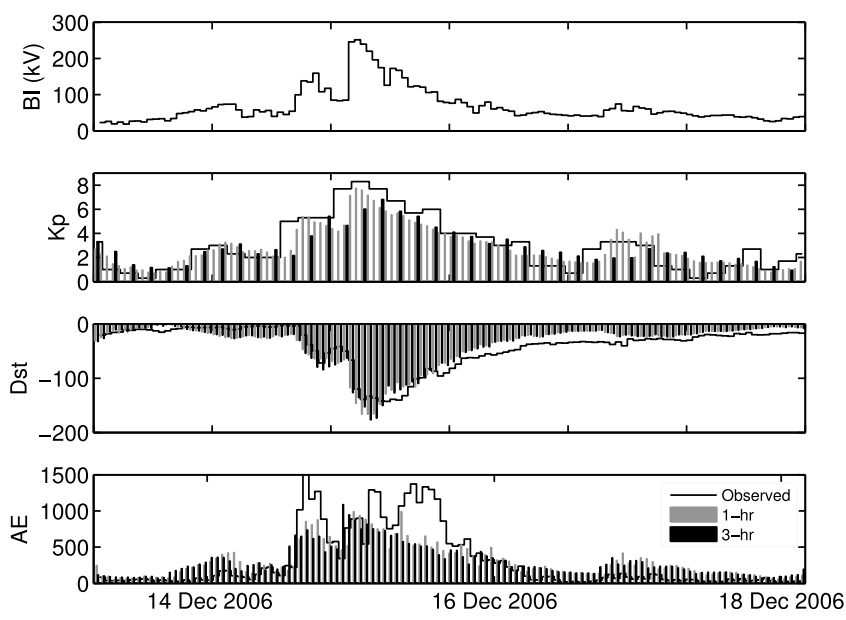

Figure 3. The $1 \mathrm{~h}$ and $3 \mathrm{~h}$ predictions from BI-only models compared with observed data for 14-18 December 2006. Dst here is compared with pressure-corrected Kyoto Dst data.

[14] A few common metrics to be used here to compare models are the linear correlation coefficient, RMSE (rootmean-square error) and ARE (average relative error). The RMSE and ARE are given by

$$
\begin{aligned}
R M S E & =\sqrt{\frac{1}{N} \sum_{t=1}^{N} \mid \text { model }_{t}-\text { observed }\left._{t}\right|^{2}} \\
A R E & =\frac{1}{N} \sum_{t=1}^{N} \frac{\mid \text { model }_{t}-\text { observed }_{t} \mid}{\mid \text { model }_{t} \mid}
\end{aligned}
$$

where observed ${ }_{t}$ is the actual desired value and model $_{t}$ is the model output. $\mathrm{N}$ is the total number of samples. As a note, the RMSE is also used for neural network optimization to track the time evolution of the network training error computed at the end of each epoch before the weights are updated. While the models are not evaluated based on the RMSE error alone, it is enough to use the RMSE for initial network diagnostics and pruning, i.e., to monitor learning histories as a function of time.

\section{1. $K p$ Prediction}

[15] In paper 1, the models were based on network trained on data set selected from 1997-2005. In this paper, with the inclusion of 2 more years of data (2008 and 2009) in the training set, we have the advantage of modeling one of the deepest solar minima; the past solar cycle 23 minimum was abnormally low in terms of activity level and long in duration [Antia and Basu, 2010]. As we continued to make improvements to our models with more data available for training and testing, the new best functions to predict the $K p$ index are given by the following: $K p_{t+1} \equiv f\left(B I_{t}, B I_{t-1}, .\right.$. , $B I_{t-8}$ and $K p_{t+3} \equiv f\left(\overline{B I}_{t}, \overline{B I}_{t-3}, \ldots, \overline{B I}_{t-21}\right)$, where ' $t$ ' represents
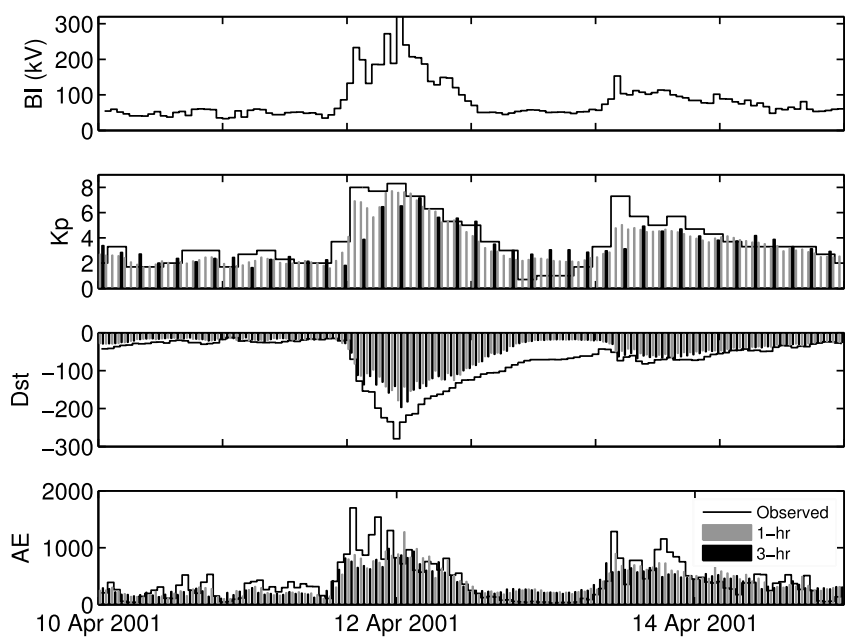

Figure 4. The $1 \mathrm{~h}$ and $3 \mathrm{~h}$ predictions from BI-only models compared with observed data for 10-14 April 2001. Dst here is compared with pressure-corrected Kyoto Dst data.

the epoch in question while ' $t-11^{\prime}, ' t+1$ ' means $1 \mathrm{~h}$ behind and $1 \mathrm{~h}$ ahead of $t$, respectively. $K p_{t+1}$ and $K p_{t+3}$ are the forecasted values, and each $B I_{t}, B I_{t-1}, B I_{t-8}$ etc. are hourly averages or $3 \mathrm{~h}$ averages of the $\mathrm{BI}$ (denoted by $\left.\overline{B I}_{t-3} \overline{B I}_{t-6}, ..\right)$, as the case may be; given that official $K p$ s are $3 \mathrm{~h}$ averages, data granularity of an hour is obtained by oversampling.

[16] To demonstrate more, we also evaluated our models retrospectively at two other magnetically active times (Dst < $-120 \mathrm{nT}$ ). The second panels of Figures 3 and 4 show how the $K p$ models performed during the December 2006 and April 2001 storms, respectively, with the corresponding BI in the top panel. In both the cases, $1 \mathrm{~h} \mathrm{Kps} \mathrm{(} \mathrm{r}=0.873$ and 0.899$)$ were slightly better predicted than $3 \mathrm{~h} \mathrm{Kps}(\mathrm{r}=0.870$ and 0.776). The overall validation results are shown in Table 1. We can notice that using the BI as a the solar wind driver, the $1 \mathrm{~h}$ model $(\mathrm{r}=0.854$; RMSE $=0.681)$ performs better than the $3 \mathrm{~h}$ model $(\mathrm{r}=0.812$; RMSE $=0.764)$. The ARE for the Kp index is set at 4 to emphasize moderate levels of activity. The last column in Table 1 shows the "significance of correlation

Table 1. Prediction Summary of the ANN-Based Models Using Only the Boyle Index ${ }^{a}$

\begin{tabular}{ccccc}
\hline Forecast Model: Using the BI & $r^{\mathrm{b}}$ & RMSE & ARE $^{\mathrm{c}}$ & $t$ Test \\
\hline$K p_{t+1}: 1 \mathrm{~h} K p$ predictor & 0.854 & 0.681 & 0.212 & $<0.1 \%$ \\
$K p_{t+3}: 3 \mathrm{~h} K p$ predictor & 0.812 & 0.764 & 0.265 & $<0.1 \%$ \\
$K p_{t+6}: 6 \mathrm{~h} \mathrm{Kp}$ predictor & 0.687 & 0.947 & 0.367 & $<0.1 \%$ \\
$D s t_{t+1}: 1 \mathrm{~h}$ Dst predictor & 0.855 & $8.836 \mathrm{nT}$ & 0.283 & $<0.1 \%$ \\
$D s t_{t+3}: 3 \mathrm{~h} \mathrm{Dst}$ predictor & 0.833 & $9.61 \mathrm{nT}$ & 0.289 & $<0.1 \%$ \\
$D s t_{t+6}: 6 \mathrm{~h} \mathrm{Dst}$ predictor & 0.777 & $11.09 \mathrm{nT}$ & 0.370 & $<0.1 \%$ \\
$A E_{t+1}: 1 \mathrm{~h} A E$ predictor & 0.828 & $95.38 \mathrm{nT}$ & 0.298 & $<0.1 \%$ \\
$A E_{t+3}: 3 \mathrm{~h} A E$ predictor & 0.782 & $96.74 \mathrm{nT}$ & 0.344 & $<0.1 \%$ \\
$A E_{t+6}: 6 \mathrm{~h} A E$ predictor & 0.621 & $121.18 \mathrm{nT}$ & 0.499 & $<0.1 \%$ \\
\hline
\end{tabular}

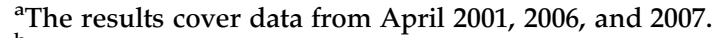

${ }^{\mathrm{b}}$ Here $r$ is the linear correlation coefficient.

${ }^{\mathrm{c}}$ Thresholds for the ARE: $K p>4, D s t<-40 \mathrm{nT}$, and $A E>500 \mathrm{nT}$. 


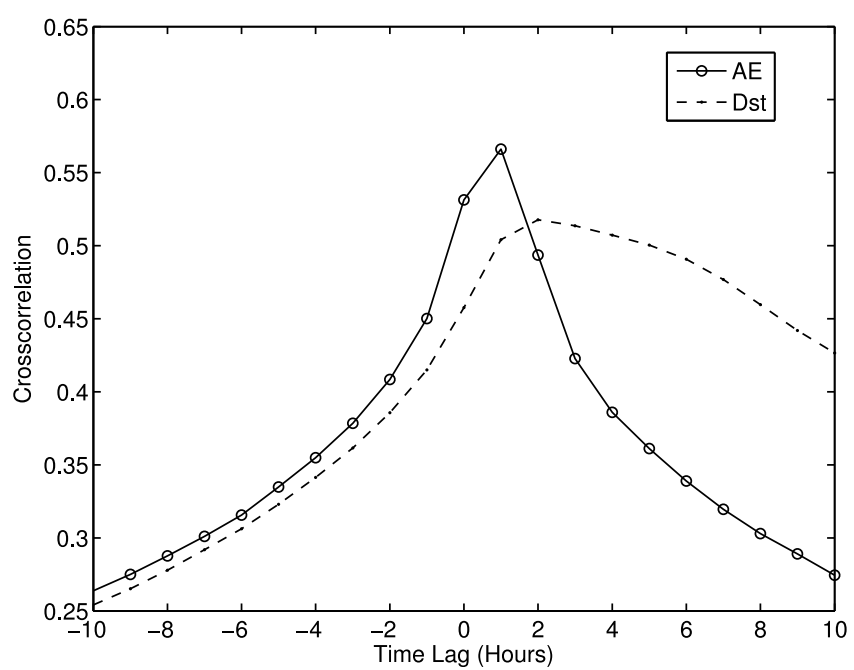

Figure 5. Cross-correlation function of BI versus $-1^{*} D s t$ and $A E$ are shown here using the raw untrained data. While the Dst tends to drop off rather slowly, the $A E$ decays rapidly, making it harder for the prediction algorithms to predict over $2 \mathrm{~h}$ ahead.

coefficients" demonstrated using statistical $t$ tests as was done in paper 1 , and clearly they are significant to below $1 \%$ probability level. Given the number of points in the distribution ( $>18000$ for $1 \mathrm{~h}$ model and $>6000$ in the case of $3 \mathrm{~h}$ model), the tests eliminate any "random" correlations for the data presented for validation.

[17] As our need for modern satellite systems and telecommunications continues to grow, space weather effects on these technologies can be mitigated with good shortterm predictions. Galactic cosmic rays, solar energetic particles, trapped high-energy particles are some possible causes for spacecraft malfunctions blamed on space weather [Baker, 2000]. Spacecraft anomalies and satellite failures have been reported due to high levels of geomagnetic activity as measured by global indices like the $K p$ [e.g., Spence et al., 1993; Choi et al., 2011]. Interestingly, such anomalies are not found to have just happened during solar maxima or during higher sunspot activities alone as Choi et al. [2011] point out in their work using reports from 95 GEO (Geostationary Earth Orbit) satellite anomalies. Our modified $K p$ prediction models can provide timely warning to operators of geostationary satellites such as those used for television broadcasting and terrestrial weather and up to 3 full hours ahead. The events shown in Figures 1, 3, and 4 demonstrate our capacity to model the activities at both extremes of solar cycle (April 2001, December 2006, and July 2009).

\subsection{Dst Prediction}

[18] The Dst index, developed by Sugiura [1964], calculated using four ground based magnetic field measurements near the equator, measures the energy in the ring current as the average depression of the horizontal component of the magnetic field around the Earth at low latitudes [Russell, 2000]. Since the measured fields are often influenced by other current systems existing in the magnetosphere [Burton et al., 1975], e.g., magnetopause currents and tail currents, it is customary to apply corrections to the Dst by removing their contributions. In this paper, a formal correction to the Dst index was applied using the BMR [Burton et al., 1975] algorithm, where $D s t^{\text {corrected }}=D s t-\mathrm{b} \sqrt{P_{s w}}+\mathrm{c} ; b=16 \mathrm{nT}(\mathrm{nPa})^{-1 / 2}$ and $c=$ $20 \mathrm{nT}$. While various values for $b$ and $c$ were given by different authors over the years, disagreements tend to exist over their precise values. Here, we will use the BMR values if the index stays below $-150 \mathrm{nT}$ and the $O^{\prime} B r i e n$ and McPherron [2000] values $(b=7.26$ and $c=11)$ otherwise. Therefore, since our models are corrected for pressure already, they do not model the "sudden impulse."

[19] After training our network, the best predicting functions for Dst can be written as: $D s t_{t+1 ; t+3} \equiv f\left(B I_{t}, B I_{t-1}\right.$, .., $\left.B I_{t-9}\right)$. Table 1 shows the overall validation results of the Dst $1 \mathrm{~h}(\mathrm{r}=0.855$; RMSE $=8.836 \mathrm{nT})$ and $3 \mathrm{~h}$ models $(\mathrm{r}=$ 0.833; RMSE = 9.61 nT). Again, we refer to Figure $3(r=0.938$ for $1 \mathrm{~h} \mathrm{Dst})$ and Figure $4(\mathrm{r}=0.939$ for $1 \mathrm{~h} \mathrm{Dst})$ for the two special cases. Our predictions in Figure 3 are in fair agreement with the observed Dst. However, in the case of the April 2001 storm, we note a good agreement in time history and recovery but a discrepancy in the magnitude between the largest predicted and observed values in Figure 4 . In Figure 5, we plot the correlation of BI versus Dst and $A E$ ( $-1^{*} D s t$ instead of $D s t$ is preferred for easy comparison). We observe that Dst has an extended response (recovery phase in the third panels of Figures 3 and 4 exemplifies this fact) but $A E$ has a short-term response to solar wind input.

[20] Presently, the $1 \mathrm{~h} \mathrm{Dst}$ index is released every hour by the World Data Center C2 for Geomagnetism, Kyoto, Japan and as provisional and final values. The US Geological Survey provides a near-real-time $1 \mathrm{~m}$ Dst index, using data from either four or three observatories from Honolulu, San Juan, Kakioka, and Hermanus, based on time domain and frequency space technique described in Gannon and Love [2011]. Our models offer true predictions of Dst index before the nowcast data become formally available.

\subsection{The $A E$ Index}

[21] The auroral electrojet " $A E$ " indices, introduced by Davis and Sugiura [1966], are used to characterize the global electrojet activity in the auroral zone and are derived from 1 min averages of northward $H$ component traces of a series of ground-based magnetometers. The $A E$ index is somewhat similar to the Dst index in terms of their availability. Reported by the World Data Center C2 for Geomagnetism, Kyoto, Japan every hour as hourly averages, it is derived based on the number of stations that are currently reporting at a given point of time, and as more information becomes available for use they are updated periodically.

[22] The following forms define the best functions describing our BI- $A E$ relationship: $A E_{t+1 ; t+3} \equiv f\left(B I_{t}, B I_{t-1}\right.$, .., $\left.B I_{t-9}\right)$. The $A E$ models share the same construction as the $K p$ and Dst models. The model summary is listed in 
Table 2. Prediction Summary of the ANN-Based Kp Models Using the Boyle Index and Dynamic Pressure, the Newell Function, and Borovsky Function ${ }^{a}$

\begin{tabular}{lcccc}
\hline \multicolumn{1}{c}{$K p$ Forecast Model } & $r^{\mathrm{b}}$ & RMSE & ARE $^{\mathrm{c}}$ & $t$ Test \\
\hline BI and Dp: $K p_{t+1}$ & 0.881 & 0.619 & 0.181 & $<0.1 \%$ \\
Newell function: $K p_{t+1}$ & 0.884 & 0.615 & 0.167 & $<0.1 \%$ \\
Borovsky function: $K p_{t+1}$ & 0.842 & 0.703 & 0.210 & $<0.1 \%$ \\
BI and Dp: $K p_{t+3}$ & 0.862 & 0.668 & 0.211 & $<0.1 \%$ \\
Newell function: $K p_{t+3}$ & 0.863 & 0.659 & 0.203 & $<0.1 \%$ \\
Borovsky function: $K p_{t+3}$ & 0.801 & 0.780 & 0.263 & $<0.1 \%$ \\
BI and Dp: $K p_{t+6}$ & 0.758 & 0.851 & 0.297 & $<0.1 \%$ \\
Newell function: $K p_{t+6}$ & 0.755 & 0.861 & 0.294 & $<0.1 \%$ \\
Borovsky function: $K p_{t+6}$ & 0.710 & 0.915 & 0.340 & $<0.1 \%$
\end{tabular}

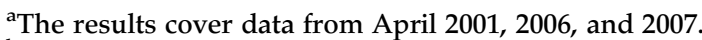

${ }^{\mathrm{b}}$ Here $r$ is the linear correlation coefficient.

${ }^{\mathrm{c}}$ Thresholds for the ARE: $K p>4, D s t<-40 \mathrm{nT}$, and $A E>500 \mathrm{nT}$.

Table $1(\mathrm{r}=0.828$ for $1 \mathrm{~h}$ and $\mathrm{r}=0.782$ for $3 \mathrm{~h}$ ). The ARE and the correlations coefficients of the $A E$ models are slightly poorer compared to the $K p$ and Dst models. Again, Figures 3 and 4 provide insights to the model performance using the two example storms. In general, the $A E^{\prime} \mathrm{s}$ response to the solar wind is rapid (steep decline BI versus $A E$ curve in Figure 5), and both of these models were trained using data of $1 \mathrm{~h}$ resolution, which tend to be rather coarse. The decline in the long-term prediction performance of the $A E$ model can also be attributed to the fact that $A E$ has two components to it, one which is directly driven by the polar cap potential, and one with a $\sim 1 \mathrm{~h}$ response time from tailside reconnection. Both depend most critically on the most recent $2 \mathrm{~h}$ of solar wind data, without an extended response, making long-term predictions more difficult.

[23] In the near future, we intend to train our algorithms using the SME index developed recently by Newell and Gjerloev [2011], an index that is a generalization of the $A E$ indices but computed from more than 100 observatories using $\sim 300$ ground-based magnetometers under the SuperMAG collaboration [Gjerloev, 2009]. This will refine our data cadence to $10 \mathrm{~min}$ or less and we are very likely to get greater specificity, but perhaps with shorter lead time. Moreover, with 100 or more observatories around the world, we can consider providing regional and local forecast of a certain index that a user might critically depend on as opposed to merely forecasting a global index.

\section{Pressure Term Inclusion: The "Ram" Functions}

[24] We have investigated and shown that the BI can best characterize the magnetospheric activity over three distinct ground-based geomagnetic indices, namely $\mathrm{K} p$, Dst, and $A E$. However, this function does not contain an explicit solar wind density term (viscous term in $v_{s w}^{2}$ and merging term in $\left.\sin ^{3}(\theta / 2)\right)$. Studies in the past [e.g., Lopez et al., 2004; Weigel, 2010] have argued the solar wind density's strong influence on the energy transfer into the magnetosphere and hence the subsequent storms and ring current activities. Borovsky et al. [1998] have shown strong correlation between the solar wind density and the density of the Earth's plasma sheet.

[25] In order to increase the baseline further, we have extended the study to accommodate the solar wind density through an explicit dynamic pressure term in the network inputs. Dynamic pressure $(D p): P_{s w}=m_{p} n_{p} v_{s w}^{2}\left(1+4 n_{a} I\right.$ $\left.n_{p}\right)$, where $n_{p}$ is the number density of the protons and $n_{a} / n_{p}$ is the alpha to proton ratio; $\mathrm{P}_{s w}$ is one of the most commonly used viscous terms besides $v_{s w}$. Here, we propose to bring an extra term into the network in the form of solar wind pressure rather than actually modifying the BI; i.e., for a $1 \mathrm{~h}$ ahead prediction model, $6 \mathrm{~h}$ of solar wind input history means having a set of $61 \mathrm{~h}$ BIs and $61 \mathrm{~h}$ Dps as inputs to the network. Unlike the original $1 \mathrm{~h}$ BI-only models, the data designated here for training, testing and validation are $\approx 47 \%, \approx 36 \%$, and $\approx 17 \%$ of the total available data. The following equations summarize the best performing functions: $K p_{t+1}^{R a m} \equiv f\left(B I_{t}, B I_{t-1}, . ., B I_{t-8} ; \sqrt[3]{D} p_{t}, \sqrt[3]{D} p_{t-1}, . ., \sqrt[3]{D} p_{t-8}\right)$; $K p_{t+3}^{\text {Ram }} \equiv f\left(\overline{B I}_{t}, \overline{B I}_{t-3}, \ldots, \overline{B I}_{t-18} ; \sqrt[3]{\overline{D p}_{t}}, \sqrt[3]{\overline{D p}_{t-3}}, . ., \sqrt[3]{\overline{D p}_{t-18}}\right)$ $[A E ; D s t]_{t+1 ; t+3}^{R a m} \equiv f\left(B I_{t}, B I_{t-1}, . ., B I_{t-9} ; \sqrt[3]{D} p_{t}, \sqrt[3]{D} p_{t-1}, . ., \sqrt[3]{D} p_{t-9}\right)$.

[26] The best performing "dynamic pressure" term for the new models is the $\sqrt[3]{D}$. We tested different powers of $D p$ before using $\sqrt[3]{D} p$. While the motivation for this approach was conceived from literature examples, our arrival to this term is merely through experimentation. The results of the new models are shown in Tables 2, 3, and 4 . Figures $6,7,8$, and 9 now show the specific cases of the December 2006 and April 2001 storms using the new models. The biggest advantage of including the dynamic pressure term in inputs can be seen in the $K p$ models. Density enhancements in the upstream solar wind due to high-speed streams following a CME are captured well through this new function, and the resulting overall statistics have improved "significantly" ( $\mathrm{r}=0.881$ vs 0.854 for $1 \mathrm{~h} ; \mathrm{r}=0.862$ vs 0.812 for $3 \mathrm{~h}$ ). However, the results from the new Dst ( $\mathrm{r}=0.861$ vs 0.855 for $1 \mathrm{~h} ; \mathrm{r}=0.840$ vs 0.833 for $3 \mathrm{~h})$ and $A E(\mathrm{r}=0.837$ vs $0.828 ; \mathrm{r}=0.802$ vs 0.782$)$ models are only slightly better than the BI-only models.

Table 3. Prediction Summary of the ANN-Based Dst Models Using the Boyle Index and Dynamic Pressure, the Newell Function, and Borovsky Function ${ }^{\mathrm{a}}$

\begin{tabular}{lcccc}
\hline \multicolumn{1}{c}{ Dst Forecast Model } & $r^{\mathrm{b}}$ & RMSE & ARE $^{\mathrm{c}}$ & $t$ Test \\
\hline BI and Dp: $D s t_{t+1}$ & 0.861 & $8.835 \mathrm{nT}$ & 0.263 & $<0.1 \%$ \\
Newell function: $D s t_{t+1}$ & 0.843 & $9.24 \mathrm{nT}$ & 0.291 & $<0.1 \%$ \\
Borovsky function: $D s t_{t+1}$ & 0.822 & $9.83 \mathrm{nT}$ & 0.293 & $<0.1 \%$ \\
BI and Dp: $D s t_{t+3}$ & 0.840 & $9.40 \mathrm{nT}$ & 0.271 & $<0.1 \%$ \\
Newell function: $D s t_{t+3}$ & 0.811 & $9.96 \mathrm{nT}$ & 0.346 & $<0.1 \%$ \\
Borovsky function: $D s t_{t+3}$ & 0.810 & $10.11 \mathrm{nT}$ & 0.300 & $<0.1 \%$ \\
BI and Dp: $D s t_{t+6}$ & 0.795 & $10.34 \mathrm{nT}$ & 0.331 & $<0.1 \%$ \\
Newell function: $D s t_{t+6}$ & 0.750 & $11.15 \mathrm{nT}$ & 0.392 & $<0.1 \%$ \\
Borovsky function: $D s t_{t+6}$ & 0.748 & $11.64 \mathrm{nT}$ & 0.360 & $<0.1 \%$ \\
\hline
\end{tabular}

${ }^{\mathrm{a}}$ The results cover data from April 2001, 2006, and 2007.

${ }^{\mathrm{b}}$ Here $r$ is the linear correlation coefficient.

${ }^{\mathrm{c}}$ Thresholds for the ARE: $K p>4, D s t<-40 \mathrm{nT}$, and $A E>500 \mathrm{nT}$. 
Table 4. Prediction Summary of the ANN-Based AE Models Using the Boyle Index and Dynamic Pressure, the Newell Function, and Borovsky Function ${ }^{\mathrm{a}}$

\begin{tabular}{lcccc}
\hline \multicolumn{1}{c}{$A E$ Forecast Model } & $r^{\mathrm{b}}$ & RMSE & ARE $^{\mathrm{c}}$ & $t$ Test \\
\hline BI and Dp: $A E_{t+1}$ & 0.837 & $92.90 \mathrm{nT}$ & 0.289 & $<0.1 \%$ \\
Newell Function: $A E_{t+1}$ & 0.834 & $93.61 \mathrm{nT}$ & 0.273 & $<0.1 \%$ \\
Borovsky Function: $A E_{t+1}$ & 0.766 & $108.86 \mathrm{nT}$ & 0.353 & $<0.1 \%$ \\
BI and Dp: $A E_{t+3}$ & 0.802 & $92.85 \mathrm{nT}$ & 0.325 & $<0.1 \%$ \\
Newell Function: $A E_{t+3}$ & 0.786 & $96.82 \mathrm{nT}$ & 0.331 & $<0.1 \%$ \\
Borovsky Function: $A e_{t+3}$ & 0.750 & $102.76 \mathrm{nT}$ & 0.362 & $<0.1 \%$ \\
BI and Dp: $A E_{t+6}$ & 0.650 & $118.21 \mathrm{nT}$ & 0.465 & $<0.1 \%$ \\
Newell Function: $A E_{t+6}$ & 0.633 & $120.73 \mathrm{nT}$ & 0.479 & $<0.1 \%$ \\
Borovsky Function: $A E_{t+6}$ & 0.631 & $120.25 \mathrm{nT}$ & 0.497 & $<0.1 \%$
\end{tabular}

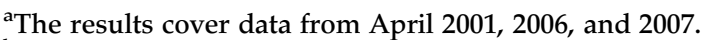

${ }^{\mathrm{b}}$ Here $r$ is the linear correlation coefficient.

'Thresholds for the ARE: $K p>4, D s t<-40 \mathrm{nT}$, and $A E>500 \mathrm{nT}$.

[27] A autocorrelation function provides another important perspective toward understanding the predicted results in that it helps distinguish a true prediction from persistence. To perform this test, we compare the autocorrelation functions of the observed time series and the predicted time series with that of itself, i.e., autocorrelation of the observed series with itself. We find that in the case of $3 \mathrm{~h}$ predictions, the cross-correlation peak (observed versus predicted) occurs at zeroth lag beating the autocorrelation (observed versus observed) at the $3 \mathrm{~h}$ lag. In this case, we have a true "successful" prediction. However, noting

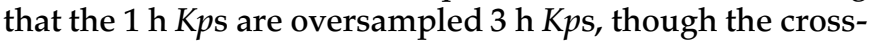
correlation function for $1 \mathrm{~h}$ predictions peaks at "zeroth" lag it falls slightly behind in value of the autocorrelation function at its $1 \mathrm{~h}$ lag. Yet, this is still an encouraging result for practical applications given the time delay or lack of availability of data in providing nowcast $K p$ s. We then examined the cross-correlation function for the Dst and $A E$
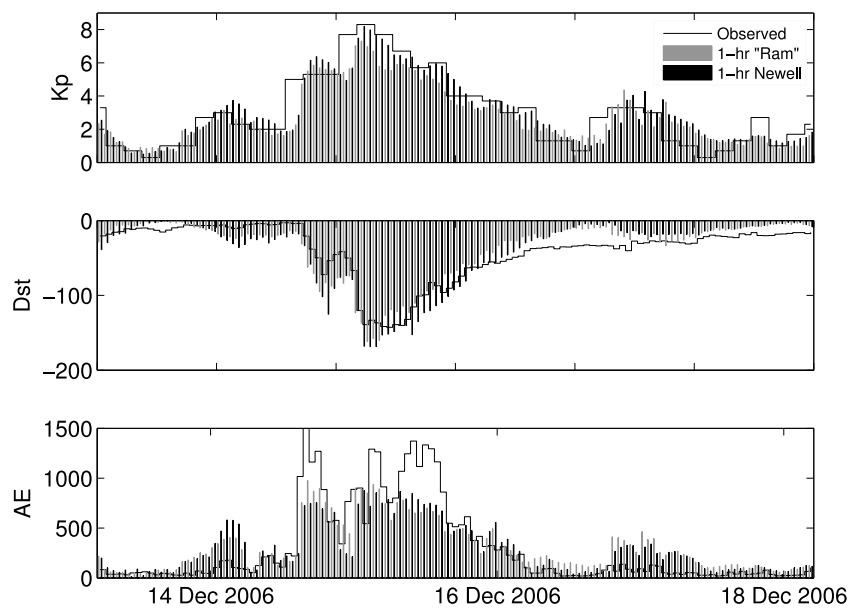

Figure 6. The $1 \mathrm{~h}$ predictions of $K p, D s t$, and $A E$ from our new models using the " $\operatorname{Ram}_{t+1}$ " functions are shown against the Newell driver for the same time period between 14 and 18 December 2006.
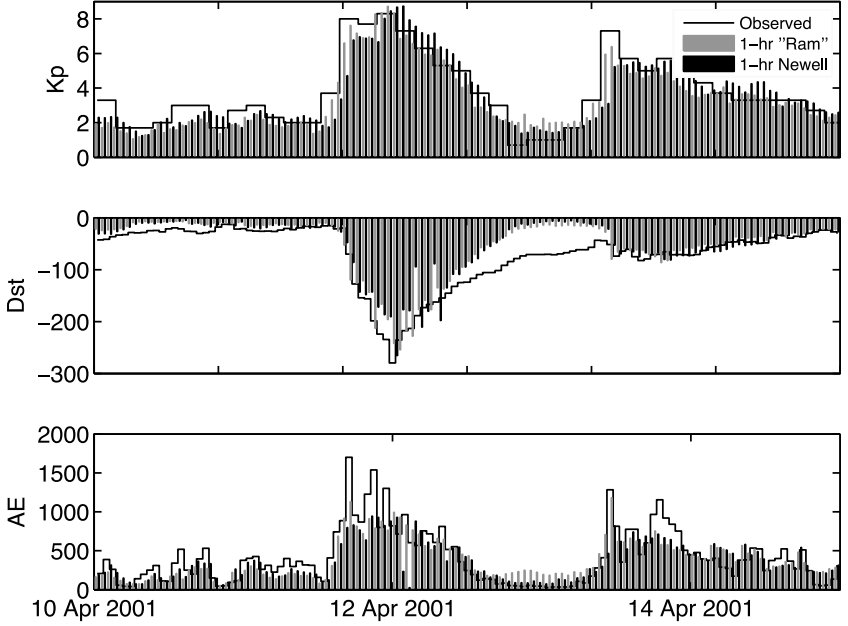

Figure 7. The $1 \mathrm{~h}$ predictions of $K p, D s t$, and $A E$ from our new models using the " $\operatorname{Ram}_{t+1}$ " functions are shown against the Newell driver for the same time period between 10 and 14 April 2001.

predictions. Our conclusion is that for the $1 \mathrm{~h}$ models of Dst and $A E$, the cross-correlation function peaks at the "zeroth" lag. However, for our $3 \mathrm{~h}$ models, the cross-correlation function peaks at negative $2 \mathrm{~h}$ lag in the case of $D s t$ and at negative $1 \mathrm{~h}$ in the case of the $A E$ index.

[28] Finally, we did not evaluate the performances of other existing models intrinsically but relied on the data found in the literature for comparisons. In fact, such one-to-one comparisons are often difficult owing to the different data sets and the time resolution used in the studies. Our new updated $\mathrm{Kp}$ models are better than the models in paper 1 $(\mathrm{r}=0.881$ vs $0.852 ; 0.862$ vs 0.845$)$, and our $1 \mathrm{~h} K p$ model is
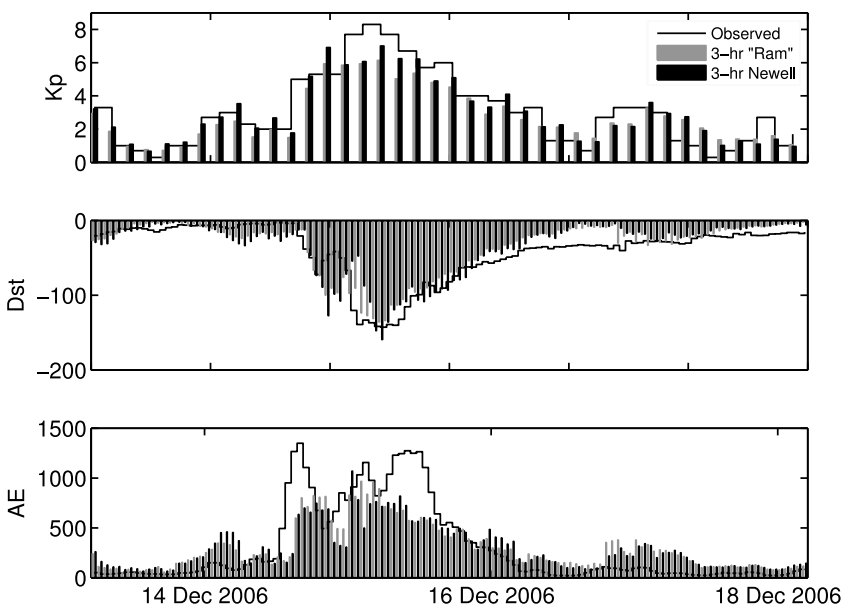

Figure 8. The $3 \mathrm{~h}$ ahead predictions of $K p$, Dst, and $A E$ from our new models using the "Ram $t+3$ " functions are shown against the Newell driver for the same time period between 14 and 18 December 2006. 

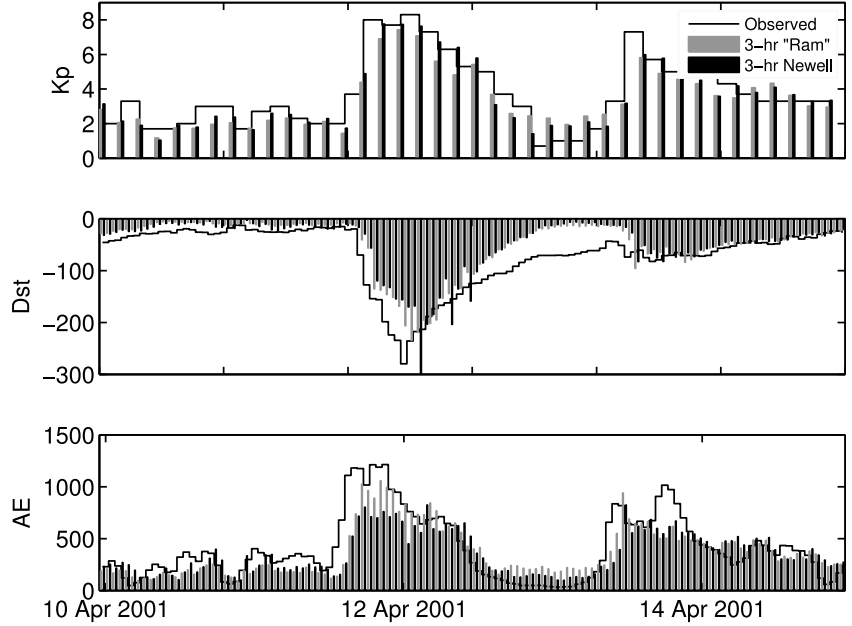

Figure 9. The $3 \mathrm{~h}$ ahead predictions of $K p, D s t$, and $A E$ from our new models using the " $\operatorname{Ram}_{t+3}$ " functions are shown against the Newell driver for the same time period between 10 and 14 April 2001.

an improvement over the APL $K p$ model $3(\mathrm{r}=0.84)$ [Wing et al., 2005]. We find our $1 \mathrm{~h}$ Dst model, with $\mathrm{r}=$ 0.861 and RMSE of $8.835 \mathrm{nT}(\approx 18200 \mathrm{~h}$ of test data) to be better than the $W u$ and Lundstedt [1997] model (RMSE = $14.7 \mathrm{nT}$ from $1957 \mathrm{~h}$ of test data), but slightly behind the Temerin and $L i$ [2002] value of $7.9 \mathrm{nT}(4320 \mathrm{~h}$ of highresolution test data). Our $3 \mathrm{~h}$ Dst model, in addition to extending the forecast lead time, performs nearly as well as our $1 \mathrm{~h}$ model $(\mathrm{r}=0.840$; $\mathrm{RMSE}=9.40 \mathrm{nT}$; $\mathrm{ARE}=0.271)$. As an example, predictions of our $1 \mathrm{~h}$ Dst model for 14-18 December 2006 is illustrated in Figure 4 with $r=$ 0.928, RMSE = $15.02 \mathrm{nT}$; real-time WINDMI Dst results [Mays et al., 2009] for the same event are $r=0.860$, RMSE $=$ $21 \mathrm{nT}$. In our future generation of Dst models, we will incorporate a longer weighting time and explore the base functions at a much higher time cadence.

\section{Newell and Borovsky Functions}

[29] A wide array of input functions can be noted in the literature, and testing them all is well beyond the scope of this paper. Therefore, we only selected two notable solar wind drivers developed recently: (a) a solar wind coupling function that is derived empirically to characterize a host of geomagnetic indices [Newell et al., 2008] and (b) a solar wind coupling function, derived from first principle, that takes into account the dayside reconnection parameters [Borovsky, 2008]. We have created two new independent sets of ANN models that are optimized for performance. In order to eliminate any bias, we have trained, tested and validated them individually before evaluating the results.

[30] Newell et al. [2008], from a rigorous analysis, have shown that the single coupling function from the solar wind is successful in predicting a wide variety of magnetospheric phenomenon without relying on the time history of the target index. Using their merging term $\left(d \Phi_{M P} / d t=v_{s w}^{4 / 3} B_{T}^{2 / 3} \sin ^{8 / 3}(\theta / 2)\right)$ alone, they were able to correlate 9 out of 10 indices of magnetospheric activity including $K p$, Dst and the $A E$ index. However, combining their top-performing viscous function $\left(n^{1 / 2} v_{s w}^{2}\right)$ with the merging term provided the best combination overall to predict up to $61 \%$ of variance across all indices. We have trained, tested and validated new ANN architectures based on input time histories of the Newell functions; separate time histories were created for both coupling and viscous functions. The best functions are explicitly given by $K p_{t+1}^{\text {Newell }} \equiv f\left(C_{t}, C_{t-1}, . ., C_{t-8} ; V_{t}, V_{t-1}, . ., C_{t-8}\right)$, $K p_{t+3}^{\text {Newell }} \equiv f\left(\bar{C}_{t}, \bar{C}_{t-3}, . ., \bar{C}_{t-18} ; \bar{V}_{t}, \bar{V}_{t-3}, . ., \bar{V}_{t-18}\right)$, and $[A E ;$ $D s t]_{t+1 ; t+3}^{\text {Newell }} \equiv f\left(C_{t}, C_{t-1}, . ., C_{t-9} ; V_{t}, V_{t-1}, . ., C_{t-9}\right)$, where ' $\mathrm{C}$ ' and ' $\mathrm{V}$ ' denotes the coupling and viscous components respectively for $1 \mathrm{~h}$ averages $(3 \mathrm{~h}$ averages are explicitly denoted by $\bar{C}$ and $\bar{V}$ ).

[31] Tables 2, 3, and 4 summarize the validation results. The Newell driver predicts slightly better in the $K p$ models ( 0.884 vs $0.881 ; 0.863$ vs 0.862$)$ and slightly worse than the "Ram" functions on the Dst and $A E$ models. The prediction performances of the Newell driver and "Ram" functions are too close that we have explicitly compared them through head-to-head tests. To be consistent, the results illustrated in Figures 6, 7, 8, and 9 are the two specific cases of storms as discussed in section 5 . It can be noted that both the models $\left(\operatorname{Ram}_{t+1}\right.$ and Newell $\left.t_{t+1}\right)$ have been able to capture the rise and fall in the activity levels. Using the same observation, we can also recognize that, in the case of Dst index, both the models are matching the peak value. We can therefore, by bringing in the viscous term, enhance the predictability of these indices. So long as there is a good measurement of the solar wind density available from an upstream monitor, one should include the viscous component as suggested in these models.

[32] Borovsky [2008] derived another coupling function R, called the solar wind "control function" (CF), by taking the dayside reconnection rate parameters into account. The control function $\mathrm{R}$ is given by the following expression: $R=$ $0.4 \mu_{o}^{1 / 2} \sin (\theta / 2) \rho_{o} V_{o}^{2}\left(1+0.5 M_{m s}^{-2}\right)\left(1+\beta_{s}\right)^{-1 / 2}\left[C \rho_{o}+(1+\right.$ $\left.\left.\beta_{s}\right)^{-1 / 2} \rho_{m}\right]^{-1 / 2}\left[1+\beta_{s}\right)^{-1 / 2}+1^{-1 / 2}$, where $\rho_{o}$ is the mass density of the solar wind upstream of the bow shock; $\mathrm{v}_{o}$ is the velocity of the solar wind upstream of the bow shock; $\mathrm{C}$ is the compression ratio of the bow shock; $\beta_{s}$ is the plasma $\beta$ value of the magnetosheath plasma near the nose; and $\mathrm{M}_{m s}$ is the magnetosonic Mach number of the solar wind. Supplements to expression $\mathrm{R}$ can be found in Borovsky [2008].

[33] The best functions that define the Borovsky driver are given by $K p_{t+1}^{\text {Borovsky }} \equiv f\left(R_{t}, R_{t-1}, . ., R_{t-7}\right) ; K p_{t+3}^{\text {Borovsky }} \equiv$ $f\left(\bar{R}_{t}, \bar{R}_{t-3}, . ., \bar{R}_{t-27}\right) ;[D s t]_{t+1}^{\text {Borovsky }} \equiv f\left(R_{t}, R_{t-1}, . ., R_{t-9}\right) ;[A E]_{t+1}^{\text {Borovsky }} \equiv$ $f\left(R_{t}, R_{t-1}, . ., R_{t-8}\right) ;[A E ; D s t]_{t+3}^{\text {Borovsky }} \equiv f\left(R_{t}, R_{t-1}, \ldots, R_{t-9}\right)$. The results of the models using the Borovsky control function is listed alongside the two coupling functions in Tables 2, 3 , and 4 . In the absence of $\mathbf{M}_{m s}$, which is often difficult to compute, we have substituted $\mathrm{M}_{A}$ for $\mathrm{M}_{m s}$ above as suggested in Borovsky [2008]. We have also assumed $\rho_{m}=0$. 

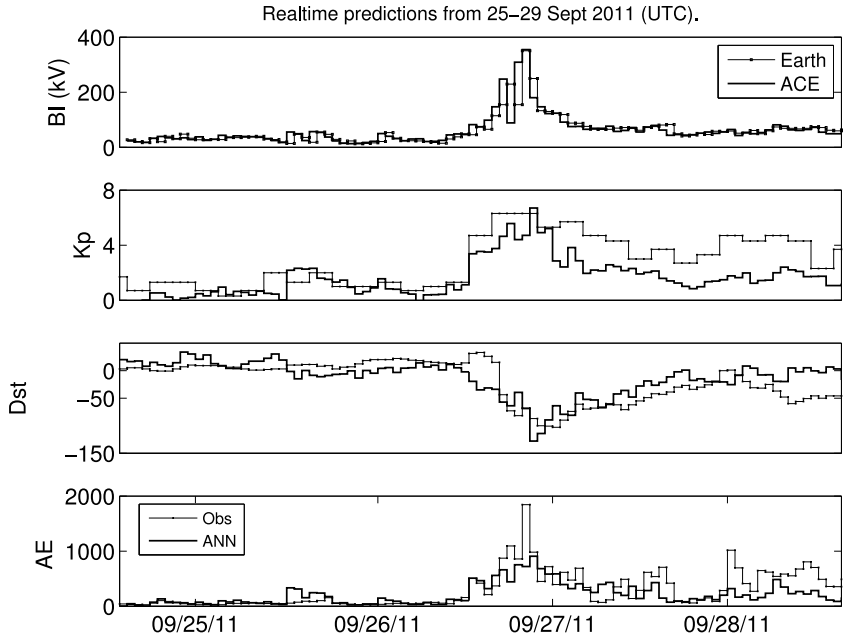

Figure 10. The $1 \mathrm{~h}$ ahead predictions of $K p$, Dst, and $A E$ from our new models $\left(\mathrm{BI}_{t+1}\right)$ are shown for a recent activity (26 September 2011). The top panel shows the $1 \mathrm{~h}$ BI values for both "real-time" ACE (smooth line) and time shifted "at Earth" measurements. The predictions are based on the real-time ACE data.

Therefore, given the nature of the expression and, perhaps, because of the difficulty in finding the right observed variables using an upstream solar wind monitor, this function is less effective as a predictor.

\subsection{Longer-Range Predictions}

[34] The two important issues relevant to space weather forecasting are accuracy and lead time. Thus far, we focused on the former, improving the accuracy of short-term forecasts in the range 1-3 h. Here we attempt to raise the lead time further. We trained the network with $3 \mathrm{~h}$ averages in order to predict $6 \mathrm{~h}$ ahead. The best functions are $K p_{t+6}^{\operatorname{Ram}} \equiv f\left(\overline{B I}_{t}, \overline{B I}_{t-3}, \ldots, \overline{B I}_{t-18} ; \sqrt[3]{\overline{\overline{D p}}_{t}}, \sqrt[3]{\overline{\overline{D p}}_{t-3}}, . ., \sqrt[3]{\overline{\overline{D p}}_{t-18}}\right)$; $[D s t]_{t+6}^{R a m} \equiv f\left(\overline{B I}_{t}, \overline{B I}_{t-3}, \ldots, \overline{B I}_{t-27} ; \sqrt[3]{\overline{D p}}, \sqrt[3]{\overline{D p}_{t-3}}, . ., \sqrt[3]{\overline{D p}_{t-27}}\right)$; $[A E]_{t+6}^{\operatorname{Ram}} \equiv F\left(\overline{B I}_{t}, \overline{B I}_{t-3}, \ldots, \overline{B I}_{t-21} ; \sqrt[3]{\overline{D p}}, \sqrt[3]{\overline{D p}_{t-3}}, . ., \sqrt[3]{\overline{D p}} t-21\right)$.

[35] Tables 2, 3, and 4 list the summary of these models using the same reference thresholds as in Table 1, which is our baseline for comparison. The best performing model is the $K p$ model followed by Dst and then the $A E$. The $A E$ index responds more rapidly to the solar wind input, so longer-range predictions are relatively difficult. Even though the prediction efficiency of all the models has dropped significantly going from $3 \mathrm{~h}$ to full $6 \mathrm{~h}$, one can use these models as a baseline for issuing probabilistic forecasts, which can be updated for accuracy as we get nearer the forecasted time.

\section{Real-Time Operations}

[36] The modified $K p$ models and new algorithms to predict $D s t$ and $A E$ have been tested against historic data for new performance standards. Currently, the BI-driven $1 \mathrm{~h}$ and $3 \mathrm{~h}$ models are up and running and the predictions can be accessed in real time at http://mms.rice.edu/ realtime/forecast.html. In addition to this, the webpage also provides the status of the current space weather condition (updating every $10 \mathrm{~min}$ ) and, as an educational resource, description of various parameters of the solar wind. Interested users can go to http://mms.rice.edu/ realtime/dials.html and to the links therein.

[37] As an example, Figure 10 shows predictions from a recent activity (26 September 2011). The activity saw high spikes in the BI, reaching a $10 \mathrm{~min}$ averaged peak value of $373 \mathrm{kV}$ on 26 September at 1836 UTC. The first panel shows the real-time estimates (without time shifts) of the BI versus the $1 \mathrm{AU} 1 \mathrm{~h}$ merged values archived at the OMNIWeb. Correspondingly, the $1 \mathrm{~h}$ predictions of $K p$, $D s t$, and $A E$ is also shown. The predictions reached alert level thresholds much earlier than the observed peak value of the BI, which occurred on Monday, 26 September at 15:46:00 UTC. A prediction-based "Yellow" alert was issued on Monday, 26 September at 15:04:00 UTC followed by another prediction-based "Red" on Monday, 26 September at 18:04:00 UTC.

[38] A newly modified version of the existing alert system to send out "alerts" and "warnings" to the subscribers of our "spacalrt," by giving key considerations to the results of this paper, is in effect. Our subscribers have been receiving notices and warnings through this new system in the event of any severe geomagnetic activity that may be ongoing or imminent. An alert is called "Yellow" and issued whenever two consecutive measurements of $10 \mathrm{~min}$ averages of the BI exceed $150 \mathrm{kV}$ or if either of the NOAA estimated planetary K index (http://www.swpc.noaa.gov/ rt_plots/kp_3d.html) or the ANN predicted $K p$ exceeds 4 . An alert is called "Red" and issued if it meets any of the following criterion: (a) the 10 min BI exceeds $200 \mathrm{kV}$, provided the previous had been at least $150 \mathrm{kV}$; (b) the NOAA estimated planetary $\mathrm{K}$ index exceeds 6; or (c) the ANN predicted $K p$ exceeds 6 , and in this case the "Red" alert could be an upgrade to a previously issued "Yellow" alert or a fresh alert by itself. Interested users can subscribe to our system by sending an e-mail to spacalrt-subscribe@ mailman.rice.edu.

\section{Conclusions}

[39] In this work, we have introduced new Dst and $A E$ and improved $K p$ prediction models based on solar wind coupling functions trained using ANNs. Not surprisingly, our analyses indicate a decline in the prediction accuracy with increasing lead time. The predictive power of the BI driven models increases with the inclusion of dynamic pressure term. We have also trained and tested the models with other notable functions found in the literature: the Newell function and the Borovsky control function. The Newell function is somewhat similar in form to the BI. While the Newell function proves to have a slight advantage over the BI for predicting $K p$, the Boyle index 
combined with the pressure term does well in predicting the Dst and $A E$ indices.

[40] The new forecast models are running in real time and the results are available at http://mms.rice.edu/ realtime/forecast.html. Our $1 \mathrm{~h}$ models are useful for applications needing accurate real time forecasts of global geomagnetic indices. This might include satellite operators, high-voltage power grid companies, oil pipe lines for whom sudden fluctuations in the geomagnetic fields could be catastrophic. We also provide forecasts with lead times of $3 \mathrm{~h}$ and $6 \mathrm{~h}$, but with slightly higher uncertainly. Currently, we are also exploring the possibility of running our models to provide longer lead times through the use of data from the STEREO Real-Time Space Weather Beacon or a suitable monitor at the L5 Lagrangian point that may become available in the future. This would help keep the "false alarm" rates to a minimum and make the models more accurate.

[41] Acknowledgments. This paper is based upon work supported partially by the William and Elva Gordon fund, NASA under cooperative agreement NAG5-316, and by the MMS mission under contract to SWRI. We thank the ACE Science Center for providing the ACE data. We also thank the OMNIWeb for maintaining the solar wind, magnetic field, and plasma data. We also wish to thank the GeoForschungsZentrum (GFZ), Potsdam, Germany for maintaining and providing the official $K p$ records and the World Data Center for Geomagnetism, Kyoto for maintaining the Dst and $A E$ records.

\section{References}

Antia, H. M., and S. Basu (2010), Solar rotation rate during the cycle 24 minimum in activity, Astrophys. J., 720(1), 494-502, doi:10.1088/0004$637 X / 720 / 1 / 494$.

Baker, D. N. (2000), The occurrence of operational anomalies in spacecraft and their relationship to space weather, IEEE Trans. Plasma Sci., 28, 2007-2016, doi:10.1109/27.902228.

Bala, R., P. H. Reiff, and J. E. Landivar (2009), Real-time prediction of magnetospheric activity using the Boyle index, Space Weather, 7, S04003, doi:10.1029/2008SW000407.

Balasubramanian, R. (2010), Forecasting geomagnetic activity indices using the Boyle index through artificial neural networks, PhD dissertation, William Marsh Rice Univ., Houston, Tex.

Boaghe, O. M., M. A. Balikhin, S. A. Billings, and H. Alleyne (2001), Identification of nonlinear processes in the magnetospheric dynamics and forecasting of Dst index, J. Geophys. Res., 106(A12), 30,047-30,066, doi:10.1029/2000JA900162.

Boberg, F., P. Wintoft, and H. Lundstedt (2000), Real time Kp predictions from solar wind data using neural networks, Phys. Chem. Earth, Part C, 25, 275-280, doi:10.1016/S1464-1917(00)00016-7.

Borovsky, J. E. (2008), The rudiments of a theory of solar wind/magnetosphere coupling derived from first principles, J. Geophys. Res., 113, A08228, doi:10.1029/2007JA012646.

Borovsky, J. E., M. F. Thomsen, and R. C. Elphic (1998), The driving of the plasma sheet by the solar wind, J. Geophys. Res., 103(A8), 17,617-17,639, doi:10.1029/97JA02986.

Boyle, C. B., P. H. Reiff, and M. R. Hairston (1997), Empirical polar cap potentials, J. Geophys. Res., 102, 111-125, doi:10.1029/96JA01742.

Burton, R. K., R. L. McPherron, and C. T. Russell (1975), An empirical relationship between interplanetary conditions and Dst, J. Geophys. Res., 80(31), 4204-4214, doi:10.1029/JA080i031p04204.

Choi, H.-S., J. Lee, K.-S. Cho, Y.-S. Kwak, I.-H. Cho, Y.-D. Park, Y.-H. Kim, D. N. Baker, G. D. Reeves, and D.-K. Lee (2011), Analysis of GEO spacecraft anomalies: Space weather relationships, Space Weather, 9, S06001, doi:10.1029/2010SW000597.

Costello, K. A. (1997), Moving the Rice MSFM into a real-time forecast mode using solar wind driven forecast models, $\mathrm{PhD}$ dissertation, William Marsh Rice Univ., Houston, Tex.
Davis, T. N., and M. Sugiura (1966), Auroral electrojet activity index $A E$ and its universal time variations, J. Geophys. Res., 71, 785-801, doi:10.1029/JZ071i003p00785.

Gannon, J. L., and J. J. Love (2011), USGS 1-min Dst index, J. Atmos. Sol. Terr. Phys., 73(2-3), 323-334, doi:10.1016/j.jastp.2010.02.013.

Gavrishchaka, V. V., and S. B. Ganguli (2001), Optimization of the neural-network geomagnetic model for forecasting large-amplitude substorm events, J. Geophys. Res., 106(A4), 6247-6257, doi:10.1029/ $2000 J A 900137$.

Gjerloev, J. W. (2009), A global ground-based magnetometer initiative, Eos Trans. AGU, 90(27), 230, doi:10.1029/2009EO270002.

Gleisner, H., and H. Lundstedt (1997), Response of the auroral electrojets to the solar wind modeled with neural networks, J. Geophys. Res., 102(A7), 14,269-14,278, doi:10.1029/96JA03068.

Gleisner, H., and H. Lundstedt (2001), Auroral electrojet predictions with dynamic neural networks, J. Geophys. Res., 106(A11), 24,541-24,549, doi:10.1029/2001JA900046.

Hairston, M. R., T. W. Hill, and R. A. Heelis (2003), Observed saturation of the ionospheric polar cap potential during the 31 March 2001 storm, Geophys. Res. Lett., 30(6), 1325, doi:10.1029/2002GL015894.

Horton, W., M. J. Mithaiwala, E. A. Spencer, and I. Doxas (2005), WINDMI: A family of physics network models for storms and substorms, in Multiscale Coupling of Sun-Earth Processes, edited by A. T. Y. Lui, Y. Kamide, and G. Consolini, pp.431-445, Elsevier, Amsterdam, doi:10.1016/B978-044451881-1/50032-0.

Kamide, Y., et al. (1998), Current understanding of magnetic storms: Storm-substorm relationships, J. Geophys. Res., 103(A8), 17,705-17,728, doi:10.1029/98JA01426.

Lindsay, G. M., C. T. Russell, and J. G. Luhmann (1999), Predictability of Dst index based upon solar wind conditions monitored inside 1 AU, J. Geophys. Res., 104(A5), 10,335-10,344, doi:10.1029/ 1999JA900010.

Lopez, R. E., M. Wiltberger, S. Hernandez, and J. G. Lyon (2004), Solar wind density control of energy transfer to the magnetosphere, Geophys. Res. Lett., 31, L08804, doi:10.1029/2003GL018780.

Lundstedt, H., and P. Wintoft (1994), Prediction of geomagnetic storms from solar wind data with the use of a neural network, Ann. Geophys., 12(1), 19-24, doi:10.1007/s00585-994-0019-2.

Mays, M. L., W. Horton, E. Spencer, and J. Kozyra (2009), Real-time predictions of geomagnetic storms and substorms: Use of the Solar Wind Magnetosphere-Ionosphere System model, Space Weather, 7, S07001, doi:10.1029/2008SW000459.

Newell, P. T., and J. W. Gjerloev (2011), Evaluation of SuperMAG auroral electrojet indices as indicators of substorms and aural power, J. Geophys. Res., 116, A12211, doi:10.1029/2011JA016779.

Newell, P. T., T. Sotirelis, K. Liou, C.-I. Meng, and F. J. Rich (2007), A nearly universal solar wind-magnetosphere coupling function inferred from 10 magnetospheric state variables, J. Geophys. Res., 112, A01206, doi:10.1029/2006JA012015.

Newell, P. T., T. Sotirelis, K. Liou, and F. J. Rich (2008), Pairs of solar wind-magnetosphere coupling functions: Combining a merging term with a viscous term works best, J. Geophys. Res., 113, A04218, doi:10.1029/2007JA012825.

O'Brien, T. P., and R. L. McPherron (2000), An empirical phase space analysis of ring current dynamics: Solar wind control of injection and decay, J. Geophys. Res., 105(A4), 7707-7719, doi:10.1029/ 1998JA000437.

Pallocchia, G., E. Amata, G. Consolini, M. F. Marcucci, and I. Bertello (2008), $A E$ index forecast at different time scales through an ANN algorithm based on L1 IMF and plasma measurements, J. Atmos. Sol. Terr. Phys., 70(2-4), 663-668, doi:10.1016/j.jastp.2007.08.038.

Papitashvili, V. O., N. E. Papitashvili, and J. H. King (2000), Solar cycle effects in planetary geomagnetic activity: Analysis of 36-year long OMNI dataset, Geophys. Res. Lett., 27, 2797-2800, doi:10.1029/ 2000GL000064.

Russell, C. T. (2000), The solar wind interaction with the Earth's magnetospehre: A tutorial, IEEE Trans. Plasma Sci., 28, 1818-1830, doi:10.1109/27.902211.

Siscoe, G. L., G. M. Erickson, B. U. Ö. Sonnerup, N. C. Maynard, J. A. Schoendorf, K. D. Siebert, D. R. Weimer, W. W. White, and G. R. Wilson (2002), Hill model of transpolar potential saturation: Comparisons with MHD simulations, J. Geophys. Res., 107(A6), 1075, doi:10.1029/2001JA000109. 
Spence, H. E., J. B. Blake, and J. F. Fennell (1993), Surface charging analysis of high-inclination, high-altitude spacecraft: Identification and physics of the plasma source region, IEEE Trans. Nucl. Sci., 40(6), 1521-1524, doi:10.1109/23.273510.

Sugiura, M. (1964), Hourly values of equatorial Dst for the IGY, Ann. Int. Geophys. Year, 35, 9-45.

Takahashi, K., B. A. Toth, and J. V. Olson (2001), An automated procedure for near-real-time Kp estimates, J. Geophys. Res., 106, 21,017-21,032, doi:10.1029/2000JA000218.

Takalo, J., and J. Timonen (1997), Neural network prediction of $A E$ data, Geophys. Res. Lett., 24(19), 2403-2406, doi:10.1029/97GL02457.

Temerin, M., and X. Li (2002), A new model for the prediction of Dst on the basis of the solar wind, J. Geophys. Res., 107(A12), 1472, doi:10.1029/2001JA007532.
Temerin, M., and X. Li (2006), Dst model for 1995-2002, J. Geophys. Res., 111, A04221, doi:10.1029/2005JA011257.

Weigel, R. S. (2010), Solar wind density influence on geomagnetic storm intensity, J. Geophys. Res., 115, A09201, doi:10.1029/ 2009JA015062.

Wing, S., J. R. Johnson, J. Jen, C.-I. Meng, D. G. Sibeck, K. Bechtold J. Freeman, K. Costello, M. Balikhin, and K. Takahashi (2005) Kp forecast models, J. Geophys. Res., 110, A04203, doi:10.1029/ 2004JA010500.

$\mathrm{Wu}$, J.-G., and H. Lundstedt (1997), Geomagnetic storm predictions from solar wind data with the use of dynamic neural networks, J. Geophys. Res., 102, 14,255-14,268, doi:10.1029/97JA00975. 